\title{
System analysis of sagittal plane human motion wearing an exoskeleton using marker technology
}

\author{
Sergey Jatsun ${ }^{1, a}$, Sergei Savin ${ }^{1}$, Boris Lushnikov ${ }^{1}$ and Andrey Yatsun ${ }^{1}$ \\ ${ }^{1}$ Southwest State University, Department of mechanics, mechatronics and robotics, 305040 Kursk, Russia
}

\begin{abstract}
This paper discusses various methods of obtaining time functions for joint angle that describe a exoskeleton's motion during sit-to-stand motion. This article demonstrates that functions obtained by solving the inverse kinematics problem can be effectively used as inputs to the control system of the robot. Comparison with experimentally data obtained using marker technology is done.
\end{abstract}

\section{Introduction}

Nowadays systems composed of two main elements- man and machine have become more common in robotics. These systems include devices called exoskeletons which are used to extend human abilities and enhance performance. The interaction of these two components determines the quality of the system as a whole.

A new generation of exoskeletons that allows a person to move even in the case of damage of the lower extremities recently came into service. In addition it becomes possible to significantly expand human capabilities when a person performs tasks that impose high demands on endurance and physical strength.

Obviously the creation of such devices is possible with a well-developed theory of the functioning of "manmachine" systems with particular emphasis being given to control. This also demands consideration of the manmachine interaction. Common problems in the theory of walking mechanisms have been developed in [1-10] and other publications. The focus of those publications is controlled walking which includes a wide range of problems such as gait generation and motion planning $[1-3,5,10]$, stability $[2,4]$ and obstacle negotiation $[8,9]$. At the same time we should note that for a normal person walking is preceded by the process of standing up. The problem of performing controllable motion when standing up is not as well studied as walking. There is a number of medical and technical papers discussing different aspects of sit-to-stand motion [11-17]. In papers [11-13] the use of robot suits that help paraplegic patients [11-12] and elderly people [13] to stand is discussed. Papers [14-15] analyze the use of fuzzy logic applications in exoskeletons performing this kind of motion with emphasis on the use of wearable sensors. Paper [17] discusses the use of an assistant robot that helps a person to stand up. In paper [18] experimental

\footnotetext{
${ }^{a}$ Corresponding author: teormeh@inbox.ru
}

data obtained from studying sit-to-stand motion of a person was shown and different methods of approximating it by smooth functions were compared.

After analysing the amount of presented work we can conclude that there hasn't been enough research in the area of experimental study of the motion of a person wearing an exoskeleton during the process of standing up as well as the analysis of methods of verification, processing and application of such data.

In this work an experimental study of a motion of a human wearing exoskeleton is carried out. We will use the data obtained from experiment discussed in the paper [18]. The measurements are done with sensors and using visual markers and camera footage to obtain certain kinematic parameters. The data is used to produce trajectories of exoskeleton parts that will drive the machine from sitting to standing position. This approach of obtaining such trajectories is an alternative to the one proposed in [19] where the trajectories were generated by solving the inverse kinematics problem. The advantage of using experimental data to generate the trajectories is the possibility of taking into account more individual features of the moving patterns of a person wearing an exoskeleton, thus making the machine more adaptive for the user.

\section{Statement of the problem}

The person's position is determined by generalized coordinates. In this paper we consider the case when a system of three generalized coordinates defines the orientation of the shin, hip and trunk. These parts of the human body execute plane-parallel motions whilst the foot remains stationary. Hence it turns out that the person's movement can be considered as that of a fourlink mechanism with one fixed link (fig. 1). 


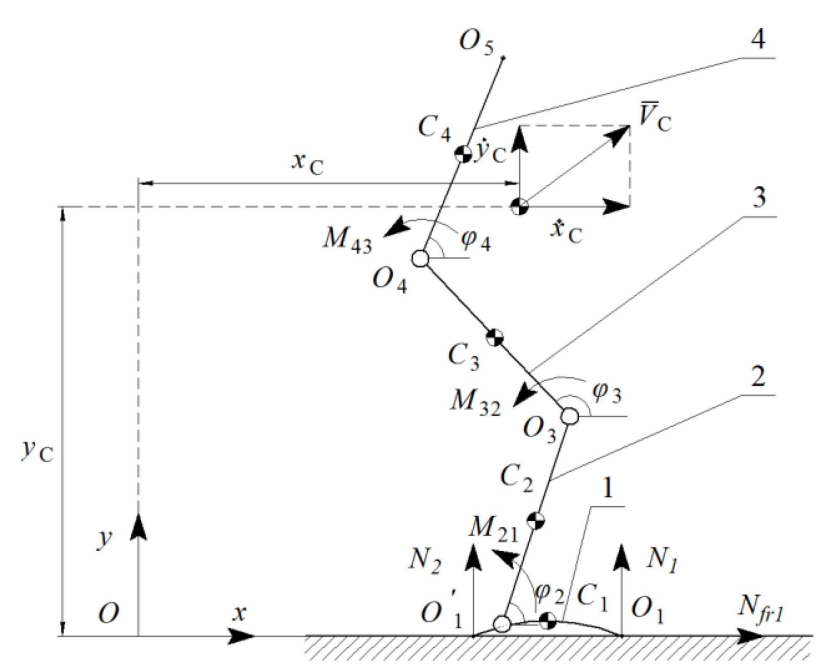

Figure 1. Four-link mechanism used to model a person wearing an exoskeleton.

The shin's orientation is determined by angle $\varphi_{2}$, the hip's orientation - by angle $\varphi_{3}$, and the trunk's orientation - by angle $\varphi_{4}$. The links have length $l_{i}$ and mass $m_{i}, i=\overline{1,4} . M_{i, i-1}$ are torques acting on the $i$-th link.

The forward kinematics of the mechanism are described by the following expressions:

$$
\begin{aligned}
& \vec{r}_{C i}^{(0)}=\left[\begin{array}{c}
x_{O 1}+\sum_{j=1}^{i-1} l_{j} \cos \left(\varphi_{j}\right)+0.5 l_{i} \cos \left(\varphi_{i}\right) \\
y_{O 1}+\sum_{j=1}^{i-1} l_{j} \sin \left(\varphi_{j}\right)+0.5 l_{i} \sin \left(\varphi_{i}\right)
\end{array}\right], \\
& \vec{r}_{O i+1}^{(0)}=\left[\begin{array}{c}
x_{O 1}+\sum_{j=1}^{i} l_{j} \cos \left(\varphi_{j}\right) \\
y_{O 1}+\sum_{j=1}^{i} l_{j} \sin \left(\varphi_{j}\right)
\end{array}\right],
\end{aligned}
$$

where $\vec{r}_{C i}^{(0)}$ and $\vec{r}_{O i}^{(0)}$ are radius vectors, describing position of points $C_{i}$ and $O_{i}$ respectively; $x_{O 1}, y_{O 1}$ coordinates that describe the position of point $O_{1}$.

Assuming $x_{O 1}=y_{O 1}=0$ the position of the center of mass $(\mathrm{CM})$ of the mechanism is described by the vector $\vec{r}_{C}^{(0)}$ (see [18]):

$\vec{r}_{C}^{(0)}=\left[\begin{array}{l}x_{C} \\ y_{C}\end{array}\right]=\left[\begin{array}{c}\sum_{i=1}^{4} K_{i} \cos \left(\varphi_{i}\right) \\ \sum_{i=1}^{4} K_{i} \sin \left(\varphi_{i}\right)\end{array}\right], K_{i}=\left(\sum_{j=i}^{4} m_{j}-0.5 m_{i}\right)\left(\sum_{j=1}^{4} m_{j}\right)^{-1} l_{i} \cdot(3)$

We will consider the case when the person's CM moves along a line described by a parametric equation during the process of standing up. Then we can solve the inverse kinematics problem and obtain expressions for $\varphi_{2}$ and $\varphi_{3}$ that drive the CM along that trajectory:

$$
\begin{aligned}
& \varphi_{2}=-\cos ^{-1}\left(\frac{\|b\|^{2}+K_{1}^{2}-K_{2}^{2}}{2 K_{1}\|b\|}\right)+\cos ^{-1}\left(\frac{b_{1}}{\|b\|}\right), \\
& \varphi_{3}=\cos ^{-1}\left(\frac{\|b\|^{2}+K_{2}^{2}-K_{1}^{2}}{2 K_{2}\|b\|}\right)+\cos ^{-1}\left(\frac{b_{1}}{\|b\|}\right), \\
& \text { where } b=\left[\begin{array}{c}
x_{C}^{*}(t)-K_{1} \cos \left(\varphi_{1}\right)-K_{4} \cos \left(\varphi_{4}\right) \\
y_{C}^{*}(t)-K_{1} \sin \left(\varphi_{1}\right)-K_{4} \sin \left(\varphi_{4}\right)
\end{array}\right], t \in\left[\begin{array}{ll}
t_{1} & t_{2}
\end{array}\right] .
\end{aligned}
$$
and $x_{C}^{*}(t), y_{C}^{*}(t)$ are functions that give parametric representation of the CM's trajectory. $\varphi_{4}$ can be chosen arbitrarily provided that for any value of $\varphi_{4}$ it should be possible to form a triangle with sides $K_{2}, K_{3}$ and $\|b\|$. We will choose $\varphi_{4}$ to be a polynomial function

$$
\varphi_{4}=\sum_{p=1}^{4} a_{p}^{(\varphi 4)} t^{p}, t \in\left[\begin{array}{ll}
t_{1} & t_{2}
\end{array}\right] .
$$

We should note that before starting the verticalization process the mechanism should relocate its center of mass such that the mechanism would be statically stable. This can be done by lowering the 4-th link. We will call this part of motion the "preliminary phase". A similar kind of motion is performed by a person when standing up from a chair. The motion of the 4-th link during preliminary phase is described by a polynomial function

$$
\varphi_{4}=\sum_{p=1}^{4} b_{p}^{(\varphi 4)} t^{p}, t \in\left[\begin{array}{ll}
t_{0} & t_{1}
\end{array}\right) .
$$

The obtained expressions are shown in fig. 2. The time interval $0<t<1 \mathrm{~s}$ corresponds to the preliminary phase.

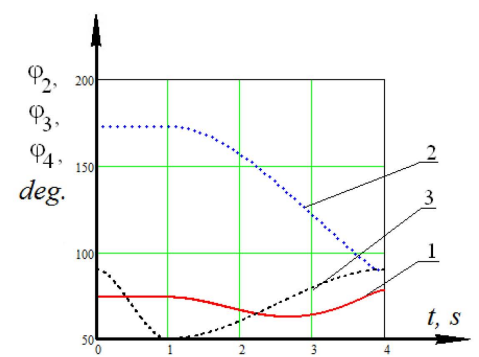

Figure 2.Graphs of $1-\varphi_{2}, 2-\varphi_{3}, 3-\varphi_{4}$.

We should note that in practice if all computations are done on the robot's on-board computer the use of expressions (4) may not be optimal. Using a spline approximations for functions $\varphi_{2}$ and $\varphi_{3}$ might speed up computations by alleviating the need for computing inverse cosines.

The dynamics of the mechanism can be described by three equations of motion: 


$$
\begin{aligned}
& \ddot{\varphi}_{2}\left(J_{C 2}+l_{2}^{2}\left(\frac{m_{2}}{4}+m_{3}+m_{4}\right)\right)+\ddot{\varphi}_{3}\left(m_{3}+2 m_{4}\right) \frac{l_{2} l_{3}}{2} \cos \left(\varphi_{3}-\varphi_{2}\right) \\
& +\ddot{\varphi}_{4} m_{4} \frac{l_{2} l_{4}}{2} \cos \left(\varphi_{4}-\varphi_{2}\right)+\dot{\varphi}_{3}^{2}\left(m_{3}+2 m_{4}\right) \frac{l_{2} l_{3}}{2} \sin \left(\varphi_{2}-\varphi_{3}\right)+ \\
& +\dot{\varphi}_{4}^{2} m_{4} \frac{l_{2} l_{4}}{2} \sin \left(\varphi_{2}-\varphi_{4}\right)= \\
& =-g \frac{l_{2}}{2}\left(m_{2}+2 m_{3}+2 m_{4}\right) \cos \left(\varphi_{2}\right)-\mu_{2} \dot{\varphi}_{2}+M_{21}-M_{23} . \\
& \ddot{\varphi}_{3}\left(J_{C 3}+m_{3} \frac{l_{3}^{2}}{4}+m_{4} l_{3}^{2}\right)+\ddot{\varphi}_{4} m_{4} \frac{l_{3} l_{4}}{2} \cos \left(\varphi_{4}-\varphi_{3}\right)+ \\
& +\ddot{\varphi}_{2}\left(m_{3}+2 m_{4}\right) \frac{l_{2} l_{3}}{2} \cos \left(\varphi_{2}-\varphi_{3}\right)+\dot{\varphi}_{2}^{2}\left(m_{3}+2 m_{4}\right) \frac{l_{2} l_{3}}{2} \sin \left(\varphi_{3}-\varphi_{2}\right)+ \\
& +\dot{\varphi}_{4}^{2} m_{4} \frac{l_{3} l_{4}}{2} \sin \left(\varphi_{3}-\varphi_{4}\right)= \\
& =-g \frac{l_{3}}{2}\left(m_{3}+2 m_{4}\right) \cos \left(\varphi_{3}\right)-\mu_{3}\left(\dot{\varphi}_{3}-\dot{\varphi}_{2}\right)+M_{32}-M_{34} . \\
& \ddot{\varphi}_{4}\left(J_{C 4}+m_{4} \frac{l_{4}^{2}}{4}\right)+\ddot{\varphi}_{3} m_{4} \frac{l_{3} l_{4}}{2} \cos \left(\varphi_{3}-\varphi_{4}\right)+ \\
& +\ddot{\varphi}_{2} m_{4} \frac{l_{2} l_{4}}{2} \cos \left(\varphi_{2}-\varphi_{4}\right)+\dot{\varphi}_{2}^{2} m_{4} \frac{l_{2} l_{4}}{2} \sin \left(\varphi_{4}-\varphi_{2}\right)+ \\
& +\dot{\varphi}_{3}^{2} m_{4} \frac{l_{3} l_{4}}{2} \sin \left(\varphi_{4}-\varphi_{3}\right)=-\frac{1}{2} g l_{4} m_{4} \cos \left(\varphi_{4}\right)-\mu_{4}\left(\dot{\varphi}_{4}-\dot{\varphi}_{3}\right)+M_{43} .
\end{aligned}
$$

From these equations we can derive expressions for torques $M_{i, i-1}$ as functions of generalized coordinates and their derivatives. The graphs of $M_{i, i-1}$ are shown in fig. 3 .

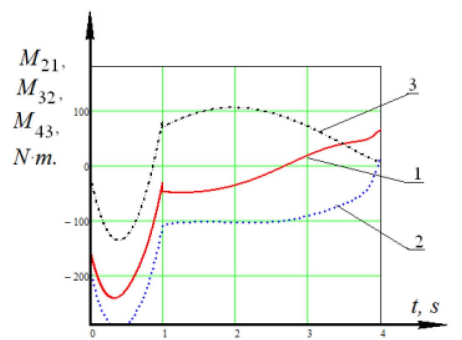

Figure 3.Graphs of $1-M_{21}, 2-M_{32}, 3-M_{43}$.

We observe that during the preliminary phase the torque graphs have a parabolic shape and after the end of this phase they rapidly change their form, producing small peaks at $t=1 \mathrm{~s}$. This peak appears because of the instantaneous change in the second derivatives of $\varphi_{2}$, $\varphi_{3}, \varphi_{4}$.

In practice it is not always possible to use the torques obtained by inverse dynamics to control a mechanical system. Small disturbances that were not taken into account when building the model can lead to loss of stability of the mechanism. To amend this feedback control is used (see fig. 4).

Here we consider a control system with a MIMO (multi input multi output) controller. Details on the implementation of this control strategy for an exoskeleton can be found in paper [18]. The graphs of $\varphi_{2}, \varphi_{3}$ and $\varphi_{4}$ obtained for the case with feedback control are shown in fig. 5 .

The graphs shown in fig. 5 are very similar to the ones shown in fig. 2. There is a slight difference during the preliminary phase. Graphs of torques $M_{i, i-1}$ generated by the control system (see fig. 6) show a more visible difference from the graphs shown in fig. 3 .

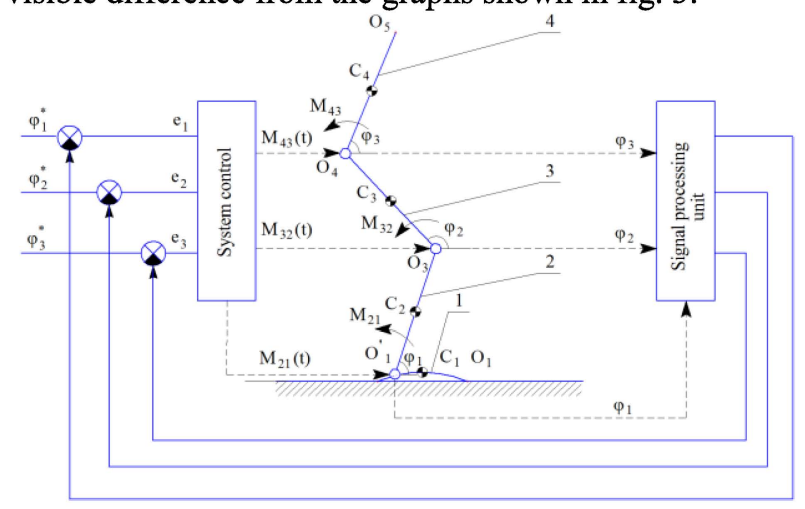

Figure 4. Mechanism with feedback control system.

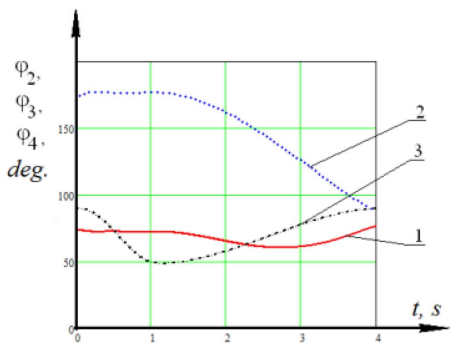

Figure 5. Graphs of $1-\varphi_{2}, 2-\varphi_{3}, 3-\varphi_{4}$ obtained for the case with feedback control.

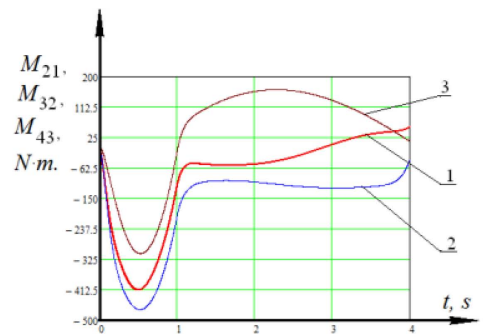

Figure 6.Graphs of $1-M_{21}, 2-M_{32}, 3-M_{43}$ obtained for the case with feedback control.

We note that the peaks observed earlier are smoothed out, while the shape of the graphs is mostly preserved. This indicates that feedback control is well suited for implementation of this type of motion.

\section{Experimental study}

In the last section we showed that the graphs of joint angles obtained by solving inverse kinematics problem and angles that come from solving equations of motion for the mechanism are similar, which suggest that it is possible to use feedback control to implement this type of motion of a four link mechanism. At the same time we should take into account the fact that the motion of the exoskeleton is performed both by the mechanism and the operator. If the motion is unnatural for the operator they will feel discomfort which may become an important issue if the person wears the exoskeleton daily.

To study the difference between the human's sit-tostand motion and the motion discussed in the previous 
section we will use experimental data derived from experiments done in the laboratory of the department of Mechanics, Mechatronics and Robotics, Southwest State University. The sensor suit can be equipped with visual markers that allow use of cameras and video processing to obtain kinematic parameters of the motion. Fig. 7 shows the human in the rising process, wearing measuring system built in the laboratory. Some details of the experiments can be found in paper [19].

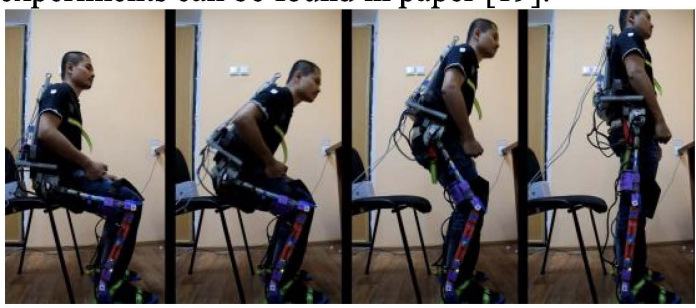

Figure 7. A person in the process of standing up.

The experimental setup shown in figure 6 consists of a seven-link mechanism that can be fixed on a human body such that the links are attached to the body parts by straps. When motion takes place in sagittal plane with the legs moving synchronously the three pairs of links move identically, which is why it is possible to use the four link mechanism as a model for this case. Each link has an accelerometer fixed on it in such a way that allows the use of the accelerometer for measuring angular displacements. Every joint is equipped with an encoder. The links strapped to the person's feet are equipped with pressure sensors.

The corresponding numerical dependences are shown in fig. 8.

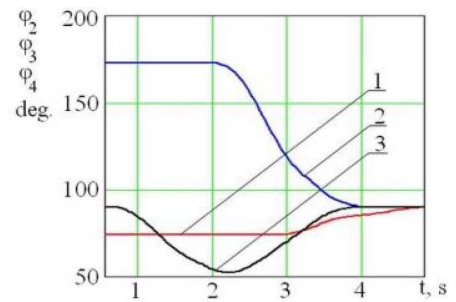

1,2 and 3 - graphs $\varphi_{2}(t), \varphi_{3}(t)$ and $\varphi_{4}(t)$, respectively

Figure 8. Experimental data after smoothing process.

The graphs shown in fig. 8 were obtained after averaging by a sliding window.

After comparing experimental data with expressions obtained by solving inverse kinematics problem we should not that the person in the experiment stands up faster, there are differences in the range of joint angles and at the end of the motion he stands up right with all angles being equal 90 degrees. The use of inverse kinematics to drive the exoskeleton into such a configuration presents great difficulty, because that is the mechanism's singular position. The difference in speed can be accounted for by changing the functions, $x_{C}^{*}(t), y_{C}^{*}(t)$ and parameter $t_{l}$.

It is possible to use experimental functions of joint angles as the control system's inputs. It is advantageous to approximate these functions by smooth functions.
In paper [19] various ways to approximate such experimentally obtained graphs by smooth functions are presented. It was shown that approximation by trigonometric polynomials has disadvantages in the constant parts of the graphs. Approximation by spline functions was shown to give more advantages.

To construct an approximation of the experimentally obtained graphs $\varphi_{2}(t), \varphi_{3}(t)$ and $\varphi_{4}(t)$ we divide functions $\varphi_{2}(t)$ and $\varphi_{3}(t)$ into three portions and $\varphi_{3}(t)$ into four sections, as was done in [19]. Let this partitioning occur at the points corresponding to the time instants: $t_{21}=3.48 \mathrm{sec}, t_{22}=4.93 \mathrm{sec}$ for the graph $\varphi_{2}(t)$, and $t_{31}=2.69 \mathrm{sec}, t_{32}=4.4 \mathrm{sec}$ for the graph, $\varphi_{3}(t)$, and $t_{41}=1.38 \mathrm{sec}, t_{42}=2.56 \mathrm{sec}, t_{43}=4.03 \mathrm{sec}$ for the graph, $\varphi_{4}(t)$. The first section and the last section of each spline are specified by a zero order polynomial and the rest is defined by a seventh order polynomials.

To calculate the polynomial coefficients, we can write the following conditions:

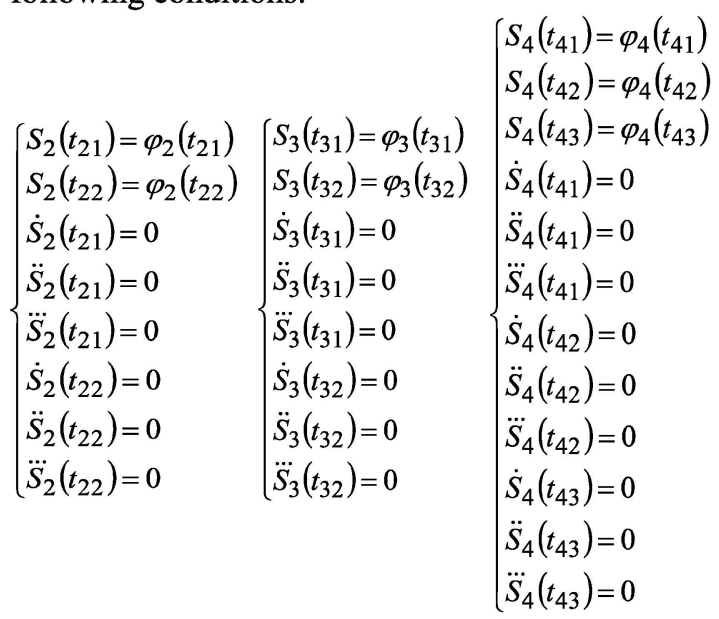

where $S_{2}(t), S_{3}(t), S_{4}(t)$ are the spline functions used to approximate the functions, $\varphi_{2}(t), \varphi_{3}(t)$ and $\varphi_{4}(t)$, respectively.

Splines that fit conditions (5) are shown in fig. 9, a). Their first and second derivatives are shown in fig. 9, b) and $\mathrm{c}$ ).

a)

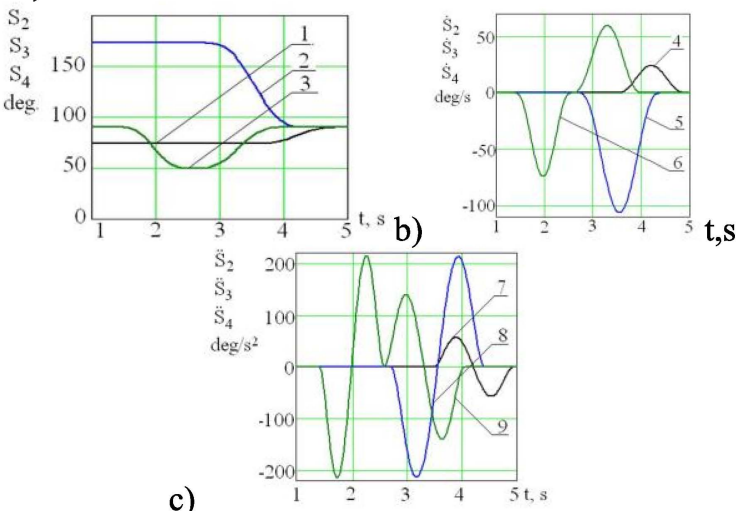

1, 2, 3 - functions $S_{2}(t), S_{3}(t), S_{4}(t), 4,5,6$ - functions $\dot{S}_{2}(t), \dot{S}_{3}(t), \dot{S}_{4}(t), 7,8,9-$ functions $\ddot{S}_{2}(t), \ddot{S}_{3}(t), \ddot{S}_{4}(t)$

Figure 9. Graphs: a) spline functions, b) first order timederivatives of spline functions, c) second order timederivatives of spline functions (see [19]). 
It is possible to achieve the absence of function discontinuities in the graphs of the time functions of generalized velocities and accelerations (fig. 9 (b) and (c)) because of the seven order spline functions.

\section{Conclusion}

This paper discusses the approach to obtain and study trajectories the sit-to-stand motion of an exoskeleton. A comparison between the trajectories obtained by solving inverse kinematics problem and the ones from solving equation of motion was carried out. It was shown that the obtained time functions of generalized coordinates can be used as an input to the feedback control system to produce the desired motion. Experimental data collected from experiments carried out at the department of Mechanics, Mechatronics and Robotics at Southwest State University was used to assess how much that motion disagrees with natural human movements during standing up process. The results of the comparison were discussed. It was noted that it is also possible to use the experimental data as input to the control system. To do that approximation by spline functions was done.

\section{Acknowledgments}

Work is performed with RSF, Project № 14-39-00008

\section{References}

1. S. Kajita, F. Kanehiro, K. Kaneko, K. Yokoi and H. Hirukawa, 2001 IEEE/RSJ International Conference on Intelligent Robots and Systems, 2001. Proceedings, 1, 239-246 (2001)

2. S. Kajita, F. Kanehiro, K. Kaneko, K. Fujiwara, K. Harada, K. Yokoi and H. Hirukawa, IEEE International Conference on Robotics and Automation, 2003. ICRA'03, 2, pp. 1620-1626 (2003).

3. K. Harada, S. Kajita, F. Kanehiro, K. Fujiwara, K. Kaneko, K. Yokoi and H. Hirukawa, IEEE/ASME Transactions on Mechatronics, 12, 53 (2007)

4. S. Kajita, M. Morisawa, K. Miura, S. Nakaoka, K. Harada, K. Kaneko and K. Yokoi, 2010 IEEE/RSJ International Conference on Intelligent Robots and Systems (IROS), pp. 4489-4496 (2010)

5. Q. Huang, K. Yokoi, S. Kajita, K. Kaneko, H. Arai, N. Koyachi and K. Tanie, IEEE Transactions on Robotics and Automation, 17, 280 (2001)

6. R. Sellaouti, O. Stasse, S. Kajita, K. Yokoi and A. Kheddar, 2006 IEEE/RSJ International Conference on Intelligent Robots and Systems, pp. 4909-4914 (2006).

7. H. Arisumi, S. Miossec, J.R. Chardonnet and $\mathrm{K}$. Yokoi, IEEE/RSJ International Conference on Intelligent Robots and Systems, IROS 2008, pp. 668675 (2008)

8. E. Yoshida, C. Esteves, T. Sakaguchi, J.P. Laumond, and K. Yokoi, 2006 IEEE/RSJ International
Conference on Intelligent Robots and Systems, pp. 827-832 (2006)

9. B. Verrelst, O. Stasse, K. Yokoi and B. Vanderborght, 2006 6th IEEE-RAS International Conference on Humanoid Robots, pp. 117-123. (2006)

10. O. Brock and O. Khatib, The International Journal of Robotics Research, 21, 1031 (2002)

11. A. Tsukahara, Y. Hasegawa and Y. Sankai, IEEE International Conference on Rehabilitation Robotics, ICORR 2009, pp. 211-217 (2009)

12. A. Tsukahara, R. Kawanishi, Y. Hasegawa and Y. Sankai, Advanced robotics, 24, 1615 (2010)

13. H.G. Jun, Y.Y. Chang, B.J. Dan, B.R. Jo, B.H. Min, H. Yang and J. Kim, 2011 IEEE International Conference on Rehabilitation Robotics (ICORR), pp. 1-5 (2011)

14. S.M. Taslim Reza, N. Ahmad, I.A. Choudhury, \& R.A.R. Ghazilla, Sensors, 14, 4342 (2014)

15. O. Salah, A.A. Ramadan, S. Sessa, A.A. Ismail, M. Fujie and A. Takanishi, International Journal of Automation and Computing, 10, 405 (2013)

16. A.M. Mughal and K. Iqbal, 2010 IEEE International Conference on Systems Man and Cybernetics (SMC), pp. 568-573 (2010).

17. A.M. López, J. Vaillant, F. Keith, P. Fraisse and A. Kheddar, 2014 14th IEEE-RAS International Conference on Humanoid Robots (Humanoids), pp. 817-822 (2014).

18. S. Jatsun, S. Savin, A. Yatsun and A. Malchikov, Advances in Robot Design and Intelligent Control, pp. 165-172 (Springer, 2016)

19. S. Jatsun, S. Savin and P. Bezmen, New Developments in Pure and Applied Mathematics, pp. 83-87 (2015) 